\title{
Diagnosis of congenital toxoplasmosis: prenatal and neonatal evaluation of methods used in Toulouse University Hospital and incidence of congenital toxoplasmosis
}

\author{
MH Bessières ${ }^{1 /+}$, A Berrebi ${ }^{2}$, S Cassaing ${ }^{1}$, J Fillaux ${ }^{1}$, JP Cambus ${ }^{1}$, A Berry ${ }^{1}$, C Assouline ${ }^{3 /+}$, \\ JM Ayoubi ${ }^{4}$, JF Magnaval ${ }^{1}$ \\ ${ }^{1}$ Department of Parasitology and Mycology, Hôpital de Rangueil ${ }^{2}$ Department of Gynaecology Obstetric Paule de Viguier ${ }^{3}$ Department of \\ Paediatric, Hôpital des Enfants, Centre Hospitalier Universitaire de Toulouse, 1 Avenue Jean Poulhès, 31059 Toulouse, France \\ ${ }^{4}$ Department Gynaecology Obstetric Hôpital Foch, Paris, France
}

The aim of this study was to determine the incidence of congenital toxoplasmosis (CT) and to assess the performances of prenatal and neonatal diagnoses. From 1994-2005, in Toulouse University Hospital, France, amniocentesis was performed on 352 pregnant women who were infected during pregnancy. All women were treated with spiramycin and pyrimethamine-sulfadoxine when prenatal diagnosis was positive. Among the 275 foetuses with follow-up, 66 (24\%) were infected. The transmission rates of Toxoplasma gondii were 7\%, 24\% and 59\% in the first, second and third trimesters, respectively. The sensitivity and specificity of PCR on amniotic fluid (AF) were $91 \%$ and 99.5\%, respectively. One case was diagnosed by mouse inoculation with AF and six cases were diagnosed by neonatal or postnatal screening. The sensitivity and specificity of PCR on placentas were $52 \%$ and $99 \%$, respectively. The sensitivity of tests for the detection of specific IgA and IgM in cord blood was 53\% and 64\%, respectively, and specificity values were $91 \%$ and $92 \%$. In conclusion, PCR performed on AF had the highest levels of sensitivity and specificity for the diagnosis of CT. This permits an early diagnosis of most cases and should be recommended.

Key words: congenital toxoplasmosis - prenatal - neonatal - diagnosis - PCR

In France, the screening program for congenital toxoplasmosis (CT) identifies foetal infection in pregnant women (prenatal diagnosis) or in newborns (neonatal diagnosis). Prenatal diagnosis is based on the detection of Toxoplasma gondii in the amniotic fluid (AF). Neonatal screening is based on the detection of parasites in the placenta and on the detection of $\operatorname{IgM}$ and $\operatorname{IgA}$ antibodies in newborns. Polymerase chain reaction (PCR) for detection of $T$. gondii DNA in AF has improved the sensitivity of prenatal diagnosis. The aim of this study was to assess the performance of biological tests used in our centre for early diagnosis in utero and during the neonatal period of CT.

\section{PATIENTS, MATERIALS AND METHODS}

Study population - From 1985-2005, in the Department of Gynaecology-Obstetrics of Toulouse University Hospital, France, amniocentesis were performed for prenatal diagnosis of toxoplasmosis on 676 pregnant women infected during pregnancy. Since 1994, PCR has been performed on AF and placentas. In this study, we compared the efficacy of PCR and mouse inoculation on 275 AF samples and 243 placentas collected after 1994 . We also assessed the value of specific $\operatorname{IgM}$ and $\operatorname{IgA}$ detection techniques in 249 sera samples obtained at birth. All women were treated with spiramycin and then with

+ Correponding author: regis.bessieres@wanadoo.fr

Received 10 October 2008

Accepted 4 December 2008 pyrimethamine-sulfadoxine $\left(\right.$ Fansidar $\left.^{\circledR}\right)$ when prenatal diagnosis was positive. Infected children were treated with pyrimethamine-sulfadoxine for one-two years. CT was defined by the persistence of specific $\mathrm{IgG}$ during the first year of life; the absence of infection was defined by a serum sample negative for specific IgG obtained from a child who was not treated during the same period.

Samples - For the prenatal diagnosis of CT, amniocentesis was performed after 18 weeks of amenorrhoea (AW) and about three-four weeks after the date of maternal infection. AF was obtained between the 18th and 39th AW.

For the neonatal diagnosis of CT, placenta and cord blood were collected. A sample of infant blood was taken on day 10 post partum when serology testing detected the presence of specific $\operatorname{IgM}$ and $\operatorname{IgA}$ antibodies in cord blood.

Detection of T. gondii - For the detection of T. gondii by mouse inoculation, AF and placenta were treated as described previously (Bessières et al. 2001).

The detection of $T$. gondii DNA was performed by PCR on AF and placenta. Extraction methods, DNA targets, primer pairs and detection methods used for this diagnosis were described previously (Bessières et al. 2002, Cassaing et al. 2006). From 1994-2002, conventional PCR ELISA (PCR ELISA DIG labelling and dig detection, Boehringer Mannheim) and the $B 1$ gene were used. Since 2002, real-time PCR has been performed on a Roche light cycler $^{\circledR}$ using fluorescence resonance energy transfer. The $B 1$ gene DNA target was used until 2002. Since 2003, the non-coding repetitive sequence called the RE sequence has been used. 
Toxoplasma serology - The presence of specific IgM antibodies in cord blood and neonatal serum was tested with the IgM immunosorbent agglutination assay (ISAGA, Biomérieux). All values greater than or equal to 3 were considered positive. Specific IgA antibodies were assayed by an immunocapture immunoenzymatic method. Positive values were defined by the manufacturer (Platelia Toxo IgA, Biorad).

\section{RESULTS}

Risk of CT according to gestational age at maternal seroconversion - Among a total of 676 women identified by prenatal screening, 325 prenatal diagnosis including PCR and mouse inoculation with AF have been performed since 1994. Two hundred seventy five foetuses with a follow-up and a clinical status were included in the analyses. Two hundred sixty-six live-born babies had a follow-up of one year or more. Sixty-six of 275 foetuses (24\%) were infected. Forty-nine cases were lost to follow-up: 25 during the first trimester (including 11 infections during the 1st month of pregnancy), 20 during the second and four during the third trimester. Maternal infection occurred during the first trimester of pregnancy in 96 cases, during the second trimester in 134 cases and during the third trimester in 46 cases. The transmission rates of $T$. gondii by trimester were $7 \%$ (7/96), $24 \%$ $(32 / 134)$ and $59 \%(27 / 46)$, respectively. Four medical terminations of pregnancy ( 2 maternal seroconversions at the 1st trimester and 2 at the 2nd) and two foetal deaths (maternal seroconversion at the 1st trimester) were due to CT. Neonatal deaths were observed in four cases, but were not due to toxoplasmosis. A total of 60 mothers received pyrimethamine-sulfadoxine when prenatal diagnosis was positive and the others were treated with spiramycin until the end of the pregnancy.

Prenatal diagnosis - $\mathrm{CT}$ was diagnosed in 60 cases by PCR on AF (22\%) and in 47 cases by mouse inoculation $(17 \%)$. One case was only detected by mouse inoculation
(Table I). PCR was positive in six foetuses that underwent therapeutic abortion or foetal deaths. PCR had the highest sensitivity. Forty-nine cases of live-born babies were lost to follow-up before congenital infection status was confirmed. None of these cases had a positive PCR or mouse inoculation. Two hundred sixty-six cases had a follow-up of one year and 246 underwent neonatal screening.

The sensitivity of PCR on AF was estimated at $91 \%$, positive predictive value (PPV) at $97 \%$, specificity at $99 \%$ and negative predictive value (NPV) at $97 \%$. Mouse inoculation had a sensitivity of $71 \%$, PPV of $100 \%$, specificity of $100 \%$ and NPV of $92 \%$. The sensitivity and specificity of PCR and mouse inoculation combinations were $92 \%$ and $99 \%$, respectively.

The assessment of PCR and mouse inoculation analysis on $\mathrm{AF}$, according to trimester of maternal seroconversion, is described in Table I.

Neonatal diagnosis - A total of 249 live-born babies were included at birth. Neonatal screening was considered positive when one or several tests were positive. At birth, the biological diagnosis of CT (including the detection of $T$. gondii in placenta by PCR, as well as mouse inoculation and serological tests) was positive in 48 of the 57 infected newborns (84\%). CT was not diagnosed in six cases by prenatal diagnosis; four cases were only identified through neonatal screening and two cases (1 without neonatal screening) with the postnatal followup. Concerning the detection of $T$. gondii in the placenta, the sensitivity of PCR was estimated at $52 \%$, PPV at $93 \%$, specificity at $99 \%$ and NPV at $88 \%$; for mouse inoculation, the sensitivity was estimated at $52 \%$, PPV at $96 \%$, specificity at $99 \%$ and NPV at $88 \%$ (Table II). When the two techniques were combined, the sensitivity was $57 \%$ and the specificity $99 \%$. The results of cord blood serology are shown in Table III. The sensitivity of $\operatorname{IgA}$ in cord blood was estimated at $53 \%$, PPV at $70 \%$, specificity at $91 \%$ and NPV at $86 \%$; the sensitivity of IgM was estimated at $64 \%$, PPV at $90 \%$, specificity at $94 \%$ and NPV at $90 \%$. When serologic techniques were

TABLE I

Assessment of polymerase chain reaction (PCR) and mouse inoculation analysis on amniotic fluid (AF) according to trimester at maternal seroconversion

\begin{tabular}{|c|c|c|c|c|c|}
\hline $\mathrm{AF}$ & All samples & $\begin{array}{c}\text { Sensitivity } \\
\%(\mathrm{n})\end{array}$ & $\begin{array}{l}\text { Specificity } \\
\% \text { (n) }\end{array}$ & $\begin{array}{l}\text { PPV } \\
\% \text { (n) }\end{array}$ & $\begin{array}{l}\text { NPV } \\
\%(n)\end{array}$ \\
\hline \multicolumn{6}{|l|}{ PCR } \\
\hline 1st trimester & 95 & $71(5 / 7)$ & $100(88 / 88)$ & 100 & 98 \\
\hline 2nd trimester & 134 & $87(28 / 32)$ & $99(101 / 102)$ & 97 & 96 \\
\hline 3rd trimester & 46 & $100(27 / 27)$ & $95(18 / 19)$ & 96 & 100 \\
\hline All trimesters & 275 & $91(60 / 66)$ & $99(207 / 209)$ & $97(60 / 62)$ & $97(207 / 213)$ \\
\hline \multicolumn{6}{|l|}{ Mouse inoculation } \\
\hline 1st trimester & 95 & $57(4 / 7)$ & $100(88 / 88)$ & 100 & $97(88 / 91)$ \\
\hline 2nd trimester & 134 & $75(24 / 32)$ & $100(102 / 102)$ & 100 & $93(102 / 110)$ \\
\hline 3rd trimester & 46 & $70(19 / 27)$ & $100(19 / 19)$ & 100 & $70(19 / 27)$ \\
\hline All trimesters & 275 & $71(47 / 66)$ & $100(209 / 209)$ & $100(47 / 47)$ & $92(209 / 228)$ \\
\hline PCR + mouse inoculation & 275 & $92(61 / 66)$ & $99(207 / 209)$ & $97(61 / 63)$ & $97(207 / 212)$ \\
\hline
\end{tabular}

NPV: negative predictive value; PPV: positive predictive value. 
combined, the sensitivity was $73 \%$ and the specificity was $90 \%$. With the combination of all tests, $T$. gondii detection in placenta and serological methods improved the sensitivity to $84 \%$ (Table III). On the other hand, each test used individually did not detect the majority of children with CT.

\section{DISCUSSION}

Our study confirms information about the risks of $\mathrm{CT}$ for infants of women who seroconvert during pregnancy. The risk of CT increases according to the time of maternal seroconversion (Dunn et al. 1999). Few women

TABLE II

Assessment of polymerase chain reaction (PCR) and mouse inoculation analysis on placenta according to trimester at maternal seroconversion

\begin{tabular}{|c|c|c|c|c|c|}
\hline Placenta & Total & $\begin{array}{c}\text { Sensitivity } \\
\%(\mathrm{n})\end{array}$ & $\begin{array}{l}\text { Specificity } \\
\%(n)\end{array}$ & $\begin{array}{l}\text { PPV } \\
\% \text { (n) }\end{array}$ & $\begin{array}{l}\text { NPV } \\
\%(n)\end{array}$ \\
\hline \multicolumn{6}{|l|}{ PCR } \\
\hline 1st trimester & 82 & $50(1 / 2)$ & $100(80 / 80)$ & $100(1 / 1)$ & $99(80 / 81)$ \\
\hline 2nd trimester & 119 & $62(18 / 29)$ & $98(88 / 90)$ & $90(18 / 20)$ & $89(88 / 99)$ \\
\hline 3rd trimester & 42 & $39(9 / 23)$ & $100(19 / 19)$ & $100(9 / 9)$ & $58(19 / 33)$ \\
\hline All trimesters & 243 & $52(28 / 54)$ & $99(187 / 189)$ & $93(28 / 30)$ & $88(187 / 213)$ \\
\hline \multicolumn{6}{|c|}{ Mouse inoculation } \\
\hline 1st trimester & 82 & $0(0 / 2)$ & $100(80 / 80)$ & $\mathrm{NC}$ & $98(80 / 82)$ \\
\hline 2nd trimester & 119 & $65(19 / 29)$ & $99(89 / 90)$ & $95(19 / 20)$ & $90(89 / 99)$ \\
\hline 3rd trimester & 42 & $39(9 / 23)$ & $100(19 / 19)$ & $100(9 / 9)$ & $58(19 / 33)$ \\
\hline All trimesters & 243 & $52(28 / 54)$ & $99(188 / 189)$ & $93(27 / 29)$ & $88(188 / 214)$ \\
\hline \multicolumn{6}{|c|}{ PCR + Mouse inoculation } \\
\hline 1st trimester & 82 & $50(1 / 2)$ & $100(80 / 80)$ & $100(1 / 1)$ & $99(80 / 81)$ \\
\hline 2nd trimester & 119 & $72(21 / 29)$ & $98(88 / 90)$ & $95(21 / 22)$ & $92(89 / 97)$ \\
\hline 3rd trimester & 42 & $39(9 / 23)$ & $100(19 / 19)$ & $100(9 / 9)$ & $58(19 / 33)$ \\
\hline All trimesters & 243 & $57(31 / 54)$ & $99(187 / 189)$ & $97(31 / 32)$ & $88(187 / 211)$ \\
\hline
\end{tabular}

NC: no calculated; NPV: negative predictive value; PPV: positive predictive value.

TABLE III

Comparison of immunologic tests at birth with the association of all techniques for the diagnosis of congenital toxoplasmosis according to trimester at maternal seroconversion

\begin{tabular}{|c|c|c|c|c|c|}
\hline Test & All samples & $\begin{array}{c}\text { Sensitivity } \\
\%(\mathrm{n})\end{array}$ & $\begin{array}{c}\text { Specificity } \\
\%(n)\end{array}$ & $\begin{array}{l}\text { PPV } \\
\%(n)\end{array}$ & $\begin{array}{l}\text { NPV } \\
\%(n)\end{array}$ \\
\hline \multicolumn{6}{|c|}{ ISAGA IgM on cord blood } \\
\hline 1st trimester & 83 & $0(0 / 3)$ & $93(74 / 80)$ & NC $0 / 6$ & $96(74 / 77)$ \\
\hline 2nd trimester & 121 & $66(19 / 29)$ & $96(88 / 92)$ & $83(19 / 23)$ & $83(88 / 98)$ \\
\hline 3rd trimester & 45 & $70(19 / 27)$ & $94(17 / 18)$ & $95(19 / 20)$ & $68(17 / 25)$ \\
\hline All trimesters & 249 & $64(38 / 59)$ & $94(179 / 190)$ & $78(38 / 49)$ & $90(179 / 200)$ \\
\hline \multicolumn{6}{|c|}{ ELISA IgA on cord blood } \\
\hline 1st trimester & 83 & $33(1 / 3)$ & $90(72 / 80)$ & $11(1 / 9)$ & $98(80 / 82)$ \\
\hline 2nd trimester & 121 & $48(14 / 29)$ & $95(87 / 92)$ & $74(14 / 19)$ & $85(87 / 102)$ \\
\hline 3rd trimester & 45 & $55(16 / 27)$ & $100(18 / 18)$ & $100(16 / 16)$ & $62(18 / 29)$ \\
\hline All trimesters & 249 & $53(31 / 59)$ & $91(172 / 190)$ & $70(31 / 44)$ & $86(177 / 205)$ \\
\hline \multicolumn{6}{|c|}{ IgM and IgA on cord blood } \\
\hline 1 st trimester & 83 & $33(1 / 3)$ & $89(71 / 80)$ & $10(1 / 10)$ & $97(71 / 73)$ \\
\hline 2nd trimester & 121 & $72(21 / 29)$ & $89(81 / 91)$ & $66(21 / 32)$ & $91(81 / 89)$ \\
\hline 3rd trimester & 45 & $78(21 / 27)$ & $94(17 / 18)$ & $95(21 / 22)$ & $74(17 / 23)$ \\
\hline All trimesters & 249 & $73(43 / 59)$ & $90(169 / 190)$ & $69(43 / 64)$ & $91(169 / 185)$ \\
\hline \multicolumn{6}{|l|}{ All tests } \\
\hline 1st trimester & 82 & $50(1 / 2)$ & $89(71 / 80)$ & $10(1 / 10)$ & $99(71 / 72)$ \\
\hline 2nd trimester & 119 & $86(25 / 29)$ & $87(78 / 90)$ & $93(25 / 27)$ & $95(78 / 82)$ \\
\hline 3rd trimester & 42 & $85(22 / 26)$ & $100(16 / 16)$ & $100(22 / 22)$ & $80(16 / 20$ \\
\hline All trimesters & 243 & $84(48 / 57)$ & $89(165 / 186)$ & $70(48 / 69)$ & $95(165 / 174)$ \\
\hline
\end{tabular}

NPV: negative predictive value; PPV: positive predictive value. 
who are infected late in pregnancy were included in this study; amniocentesis is rarely performed during the third trimester of pregnancy. The different methods used for the prenatal and neonatal diagnosis of CT do not yield the same results. Prenatal diagnosis using PCR on AF has considerably improved the prognosis and outcome of infected children. It is more useful than neonatal screening because almost all infected children are identified by PCR on AF. Appropriate treatment can thus be started earlier and serious complications in the foetus can be prevented. It was proven that prenatal treatment within 4 weeks of seroconversion reduced the risk of intracranial lesions compared with no treatment (Gras et al. 2005). A negative result from PCR performed on AF cannot rule out congenital infection. In this case, continuation of treatment with spiramycin and neonatal and postnatal follow-up are justified. Molecular diagnostic tests, based upon PCR using AF, have become essential in this diagnosis. The rate of false negatives is variable according to the centres. In our centre, the sensitivity was around $90 \%$, higher than in other centres (Cassaing et al. 2006, Thalib et al. 2005). The false negative must be due to a delay in transplacental transmission of $T$. gondii. During the period of this study, quantitative real-time PCR has been introduced and the PCR has been standardized.

At birth, the sensitivity of PCR and mouse inoculation in detecting $T$. gondii in placenta was lower than that of analyses using AF. The performances of these two tests were, however, similar. The poor performances of certain tests may be due to maternal treatment with pyrimethamine-sulfadoxine as observed previously (Bessières et al. 2001, Fricker Hidalgo et al. 2007). However, sensitivity of PCR performed on placenta is higher than in a recent series reported by Fricker Hidalgo et al. 2007; $\operatorname{IgA}$ or IgM testing detected $53-64 \%$ of infected infants. Sensitivity and specificity of Toxoplasma serology differ according to the nature of the study (Gilbert et al. 2007). Concerning the use of immunologic methods, the poor performances of tests for the detection of specific IgM and IgA is probably due to the fact that antibody production may have ceased at birth. On the other hand, it was not observed that treatment with pyrimethaminesulphonamide significantly reduced $\operatorname{IgM}$ and IgA sensitivity (Bessières et al. 2001, Gilbert et al. 2007).

To conclude, PCR performed on AF had the highest level of sensitivity and specificity for the diagnosis of CT. It permits an early diagnosis of most cases and should be recommended for identification of congenital infection. However, negative results cannot rule out foetal infection. Neonatal and postnatal screening identified these cases.

\section{REFERENCES}

Bessières MH, Berrebi A, Rolland M, Bloom MC, Roques C, Cassaing S, Courjault C, Séguéla JP 2001. Neonatal screening for CT in a cohort of 165 women infected during pregnancy and influence of in utero treatment on the results of neonatal tests. Eur J Obstet Gynecol Reprod Biol 94: 37-45.

Bessières MH, Cassaing S, Berrebi A, Séguéla JP 2002. Apport des techniques de biologie moléculaire dans le diagnostic prénatal de la toxoplasmose congénitale. IBS 17: 358-362.

Cassaing S, Bessières MH, A Berry A, Berrebi A, Fabre R, Magnaval JF 2006. Comparison between two amplification sets for molecular diagnosis of toxoplasmosis by real-time PCR. J Clin Microbiol 44: 720-724.

Dunn D, Wallon M, Peyron F, Petersen E, Peckham C, Gilbert G 1999. Mother-to-child transmission of toxoplasmosis: risk estimates for clinical counselling. Lancet 353: 1829-1833.

Fricker-Hidalgo H, Brenier-Pinchart MP, Schaal JP, Equy V, Bost-Bru C, Pelloux H 2007. Value of $T$. gondii detection in one hundred thirty-three placentas for the diagnosis of congenital toxoplasmosis. Pediatr Infect Dis J 26: 845-846.

Gilbert RE, Thalib L, Paul M, Wallon M, Petersen E, European Multicentre Study on CT 2007. Screening for CT: accuracy of immunoglobulin $\mathrm{M}$ and immunoglobulin A tests after birth. $J$ Med Screen 14: 8-13.

Gras L, Wallon M, Pollak A, Cortina-Borja M, Evengard B, Hayde M, Petersen E, Gilbert R, European Multicenter Study on CT 2005. Association between prenatal treatment and clinical manifestations of CT in infancy: a cohort study in 13 European centres. Acta Paediatr 94: 1721-1731.

Thalib L, Gras L, Romand S, Prusa A, Bessières MH, Petersen E, Gilbert RE 2005. Prediction of CT by polymerase chain reaction analysis of AF. BJOG 112: 567-574. 